\title{
A NOTE ON DIMENSION OF TRIANGULATED CATEGORIES
}

\author{
HIROYUKI MINAMOTO
}

(Communicated by Birge Huisgen-Zimmermann)

\begin{abstract}
In this note we study the behavior of the dimension of the perfect derived category $\operatorname{Perf}(A)$ of a dg-algebra $A$ over a field $k$ under a base field extension $K / k$. In particular, we show that the dimension of a perfect derived category is invariant under a separable algebraic extension $K / k$. As an application we prove the following statement: Let $A$ be a self-injective algebra over a perfect field $k$. If the dimension of the stable category $\underline{\bmod } A$ is 0 , then $A$ is of finite representation type. This theorem is proved by M. Yoshiwaki in the case when $k$ is an algebraically closed field. Our proof depends on his result.
\end{abstract}

\section{INTRODUCTION}

In 3. R. Rouquier introduced the dimension of triangulated categories and showed that it gives an upper bound or a lower bound of other dimensions in algebraic geometry or in representation theory (see also [4]). The dimension of triangulated categories is studied by many researchers.

In this note we study the behavior of the dimension of the perfect derived category $\operatorname{Perf}(A)$ of a dg-algebra $A$ over a field $k$ under a base field extension $K / k$. For a field extension $K / k$, we denote $A \otimes_{k} K$ by $A_{K}$.

\section{Theorem 1.1.}

(1) For an algebraic extension $K / k$, we have

$$
\text { tridim } \operatorname{Perf}(A) \leq \operatorname{tridim} \operatorname{Perf}\left(A_{K}\right) .
$$

(2) If moreover $K / k$ is separable, then equality holds.

As an application we prove the following theorem, which gives evidence that dimension of triangulated categories captures some representation theoretic properties.

The stable category $\bmod A$ plays an important role in the study of a self-injective algebra $A$ (cf. [2, 4]). If a self-injective algebra $A$ is of finite representation type, then the dimension of the stable category $\underline{\bmod } A$ is zero. Then a natural question arises as to whether the converse should also hold.

Theorem 1.2. Let $A$ be a self-injective finite dimensional algebra over a perfect field $k$. If tridim $\underline{\bmod } A=0$, then $A$ is of finite representation type.

In the case when $k$ is an algebraically closed field, this theorem is proved by M. Yoshiwaki in [5]. Our proof depends on his result.

\footnotetext{
Received by the editors April 25, 2011 and, in revised form, October 12, 2011 and February 22, 2012.

2010 Mathematics Subject Classification. Primary 16G60, 18E30.
} 


\section{Proof of Theorem 1.1}

We recall the definition of the dimension of triangulated categories. Let $\mathcal{T}$ be a triangulated category. For a full subcategory $\mathcal{I}$ of $\mathcal{T}$ we denote by $\langle\mathcal{I}\rangle$ the smallest full subcategory of $\mathcal{T}$ containing $\mathcal{I}$ which is closed under taking shifts, finite direct sums, direct summands and isomorphisms. For full subcategories $\mathcal{I}$ and $\mathcal{J}$ of $\mathcal{T}$ we denote by $\mathcal{I} * \mathcal{J}$ the full subcategory of $\mathcal{T}$ consisting of those objects $M \in \mathcal{T}$ such that there exists an exact triangle $I \rightarrow M \rightarrow J \stackrel{[1]}{\longrightarrow}$ with $I \in \mathcal{I}$ and $J \in \mathcal{J}$. Set $\mathcal{I} \diamond \mathcal{J}:=\langle\mathcal{I} * \mathcal{J}\rangle$. For $n \geq 1$ we define inductively

$$
\langle\mathcal{I}\rangle_{n}:= \begin{cases}\langle\mathcal{I}\rangle & \text { for } n=1 \\ \langle\mathcal{I}\rangle \diamond\langle\mathcal{I}\rangle_{n-1} & \text { for } n \geq 2\end{cases}
$$

Now we define the dimension of a triangulated category $\mathcal{T}$ to be

$$
\text { tridim } \mathcal{T}:=\min \left\{n \mid\langle E\rangle_{n+1}=\mathcal{T} \text { for some } E \in \mathcal{T}\right\} \text {. }
$$

To prove Theorem 1.1 we prepare some notation. We fix a field $k$. In the sequel the term $\mathrm{dg}-A$-modules means right $\mathrm{dg}-A$-modules. We denote by $C(A)$ the category of $\operatorname{dg}-A$-modules. There is an adjoint pair

$$
-\bigotimes_{A} A_{K}=-\bigotimes_{k} K: C(A) \rightleftarrows C\left(A_{K}\right): \operatorname{Hom}_{A_{K}}\left(A_{K},-\right)=: U_{K / k} .
$$

We denote by $\mathcal{D}(A)$ the derived category of $\operatorname{dg}-A$-modules. The functors $-\bigotimes_{k} K$ and $U_{K / k}$ preserve quasi-isomorphisms. Hence we obtain an adjoint pair

$$
-\bigotimes_{k} K: \mathcal{D}(A) \rightleftarrows \mathcal{D}\left(A_{K}\right): U_{K / k}
$$

If there seems to be no ambiguity, we denote $U_{K / k}$ by $U$.

We denote by $\operatorname{Perf}(A)$ the perfect derived category of $A$. This is the full triangulated subcategory of $\mathcal{D}(A)$ consisting of objects $M \in \mathcal{D}(A)$ obtained from $A \in \mathcal{D}(A)$ by taking shifts, finite direct sums, direct summands, isomorphisms and cones: $\operatorname{Perf}(A):=\bigcup_{n \geq 1}\langle A\rangle_{n}$. For $M \in \mathcal{D}(A)$ we denote by add $M$ the smallest full subcategory of $\mathcal{D}(A)$ containing $M$ which is closed under taking finite direct sums and direct summands.

Lemma 2.1. If $K / k$ is a finite dimensional extension, then for a $d g$-A-module $M$, we have add $M=$ add $U\left(M \otimes_{k} K\right)$ in $\mathcal{D}(A)$. Therefore we have $\langle M\rangle=\left\langle U\left(M \otimes_{k} K\right)\right\rangle$. Hence we have $\langle M\rangle_{n}=\left\langle U\left(M \otimes_{k} K\right)\right\rangle_{n}$ for $n \geq 1$.

Proof. The functor $U$ is a forgetful functor. Hence we have a isomorphism $U\left(M \otimes_{k}\right.$ $K) \cong M^{\oplus \operatorname{dim}_{k} K}$ in $\mathcal{D}(A)$.

Lemma 2.2. Let $K / k$ be an algebraic extension and $E$ an object of $\mathcal{D}(A)$.

(1) If an object $\bar{G}$ of $\mathcal{D}\left(A_{K}\right)$ belongs to $\left\langle E \otimes_{k} K\right\rangle_{n}$, then there exists an intermediate field $k \subset K_{0} \subset K$ which is finite dimensional over $k$ such that there exists an object $G^{\prime}$ of $\left\langle E \otimes_{k} K_{0}\right\rangle_{n}$, such that $G^{\prime} \otimes_{K_{0}} K \cong \bar{G}$ in $\mathcal{D}\left(A_{K}\right)$.

(2) Let $G$ be an object of $\mathcal{D}(A)$. If $G \otimes_{k} K$ belongs to $\left\langle E \otimes_{k} K\right\rangle_{n}$, then there exists an intermediate field $k \subset K_{0} \subset K$ which is finite dimensional over $k$ such that $G \otimes_{k} K_{0}$ belongs to $\left\langle E \otimes_{k} K_{0}\right\rangle_{n}$.

In the sequel overlined objects and morphisms are those of $\mathcal{D}\left(A_{K}\right)$, and objects and morphisms with dashes are those of $\mathcal{D}\left(A_{K_{0}}\right)$. 
Proof. (1) First note that for $\operatorname{dg}-A$-modules $M, N$ we have a natural isomorphism of Hom-spaces

$$
\operatorname{Hom}_{\mathcal{D}\left(A_{K}\right)}\left(M \otimes_{k} K, N \otimes_{k} K\right) \cong \operatorname{Hom}_{\mathcal{D}(A)}(M, N) \otimes_{k} K .
$$

It is easy to see that the problem is reduced to the following two cases, $(a),(b)$, by induction.

(a) Let $E_{1}, E_{2}$ be objects of $\mathcal{D}(A)$ such that we have an exact triangle

$$
E_{1} \otimes_{k} K \stackrel{\bar{f}}{\rightarrow} E_{2} \otimes_{k} K \rightarrow \bar{G} \stackrel{[1]}{\rightarrow}
$$

in $\mathcal{D}\left(A_{K}\right)$. By the above remark there are $f_{1}, \ldots, f_{n} \in \operatorname{Hom}_{\mathcal{D}(A)}\left(E_{1}, E_{2}\right)$ and $\alpha_{1}, \ldots, \alpha_{n} \in K$ such that we have $\bar{f}=\sum_{i=1}^{n} f_{i} \alpha_{i}$. Set $K_{0}:=k\left(\alpha_{1}, \ldots, \alpha_{n}\right)$ and $f^{\prime}:=\sum_{i=1}^{n} f_{i} \alpha_{i} \in \operatorname{Hom}_{\mathcal{D}\left(A_{K_{0}}\right)}\left(E_{1} \otimes_{k} K_{0}, E_{2} \otimes_{k} K_{0}\right)$. Then $f^{\prime} \otimes_{K_{0}} K=\bar{f}$. Therefore we have an isomorphism $G^{\prime} \otimes_{K_{0}} K \cong \bar{G}$ in $\mathcal{D}\left(A_{K}\right)$ where $G^{\prime}:=\mathrm{c}\left(f^{\prime}\right)$ is the cone of $f^{\prime}$.

(b) Let $E_{1}$ be an object of $\mathcal{D}(A)$. Assume that $\bar{G}$ is a direct summand of $E_{1} \otimes_{k} K$. Let $\bar{e}$ be an idempotent endomorphism of $E_{1} \otimes_{k} K$ which corresponds to $\bar{G}$, i.e., $\bar{e}: E_{1} \otimes_{k} K \rightarrow \bar{G} \hookrightarrow E_{1} \otimes_{k} K$, where the left arrow is the canonical projection and the right arrow is the canonical injection. Note that we can obtain the direct summand $\bar{G}$ from the corresponding idempotent endomorphism $\bar{e}$ in the following way: We define a morphism $\bar{X}:\left(E_{1} \otimes_{k} K\right)^{\oplus \mathbb{N}} \rightarrow\left(E_{1} \otimes_{k} K\right)^{\oplus \mathbb{N}}$ to be the morphism which is represented by the matrix given by

$$
\bar{X}=\left(\begin{array}{ccccc}
1-\bar{e} & 0 & 0 & 0 & \cdots \\
\bar{e} & 1-\bar{e} & 0 & 0 & \cdots \\
0 & \bar{e} & 1-\bar{e} & 0 & \cdots \\
0 & 0 & \bar{e} & 1-\bar{e} & \cdots \\
\vdots & \vdots & \vdots & \vdots & \ddots
\end{array}\right):\left(E_{1} \otimes_{k} K\right)^{\oplus \mathbb{N}} \rightarrow\left(E_{1} \otimes_{k} K\right)^{\oplus \mathbb{N}} .
$$

Then the cone $\mathrm{c}(\bar{X})$ of $\bar{X}$ is isomorphic to $\bar{G}$ in $\mathcal{D}\left(A_{K}\right)$. By the same method as above, we see that there is an intermediate field $k \subset K_{0} \subset K$ which is finite dimensional over $k$ such that there is an idempotent endomorphism $e^{\prime}$ of $E_{1} \otimes_{k} K_{0}$ such that we have $e^{\prime} \otimes_{K_{0}} K=\bar{e}$. Hence we obtain an endomorphism $X^{\prime}$ of $\left(E_{1} \otimes_{k}\right.$ $\left.K_{0}\right)^{\oplus \mathbb{N}}$ such that $X^{\prime} \otimes_{k} K_{0}=\bar{X}$. Then $\bar{G}$ is isomorphic to $G^{\prime} \otimes_{k} K_{0}$ in $\mathcal{D}\left(A_{K}\right)$, where $G^{\prime}:=c\left(X^{\prime}\right)$ is a cone of $X^{\prime}$. We see that $G^{\prime}$ is a direct summand of $E_{1} \otimes_{k} K_{0}$ by [1, Proposition 3.2.].

(2) By (1) there exists an intermediate field $k \subset K_{0} \subset K$ which is finite dimensional over $k$ such that $G^{\prime} \otimes_{K_{0}} K \cong G \otimes_{k} K$ in $\mathcal{D}\left(A_{K}\right)$ for some object $G^{\prime}$ of $\left\langle E \otimes_{k} K_{0}\right\rangle_{n}$. Let $\bar{f}: G^{\prime} \otimes_{K_{0}} K \cong\left(G \otimes_{k} K_{0}\right) \otimes_{K_{0}} K$ be an isomorphism in $\mathcal{D}\left(A_{K}\right)$. By the same method as in the proof of (1), we see that there exists an intermediate field $K_{0} \subset K_{1} \subset K$ which is finite dimensional over $k$ such that there exists an isomorphism $f^{\prime}: G^{\prime} \otimes_{K_{0}} K_{1} \cong\left(G \otimes_{k} K_{0}\right) \otimes_{K_{0}} K_{1} \cong G \otimes_{k} K_{1}$. Hence $G \otimes_{k} K_{1}$ belongs to $\left\langle E \otimes_{k} K_{1}\right\rangle_{n}$.

Proof of Theorem 1.1. (1) First we prove the case when $K / k$ is a finite dimensional extension. Assume that there exists an object $\bar{E}$ of $\operatorname{Perf}\left(A_{K}\right)$ such that $\langle\bar{E}\rangle_{n}=$ $\operatorname{Perf}\left(A_{K}\right)$. Since $K / k$ is a finite $k$ dimensional extension, $U(\bar{E})$ belongs to $\operatorname{Perf}(A)$. It is enough to prove that $\langle U(\bar{E})\rangle_{n}=\operatorname{Perf}(A)$. Let $G$ be an object of $\operatorname{Perf}(A)$. Then $G \otimes_{k} K$ belongs to $\operatorname{Perf}\left(A_{K}\right)$. Therefore $G \otimes_{k} K$ belongs to $\langle\bar{E}\rangle_{n}$. Since $U$ is exact, $U\left(G \otimes_{k} K\right)$ belongs to $\langle U(\bar{E})\rangle_{n}$. By Lemma 2.1, $G$ belongs to $\langle U(\bar{E})\rangle_{n}$. 
Next we prove the general case. Assume that there exists an object $\bar{E}$ of $\operatorname{Perf}\left(A_{K}\right)$ such that $\langle\bar{E}\rangle_{n}=\operatorname{Perf}\left(A_{K}\right)$. By Lemma 2.2, there exists an intermediate field $k \subset K_{0} \subset K$ which is finite dimensional over $k$ such that there exists an object $E^{\prime}$ of $\operatorname{Perf}\left(A_{K_{0}}\right)$ such that $E^{\prime} \otimes_{K_{0}} K \cong \bar{E}$. By the first step, it is enough to prove that $\left\langle E^{\prime}\right\rangle_{n}=\operatorname{Perf}\left(A_{K_{0}}\right)$. Let $G^{\prime}$ be an object of $\operatorname{Perf}\left(A_{K_{0}}\right)$. Since $G^{\prime} \otimes_{K_{0}} K$ belongs to $\operatorname{Perf}\left(A_{K}\right)=\left\langle E^{\prime} \otimes_{K_{0}} K\right\rangle_{n}$, by Lemma 2.2 there exists an intermediate field $K_{0} \subset K_{1} \subset K$ which is finite dimensional over $k$ such that $G^{\prime} \otimes_{K_{0}} K_{1}$ belongs to $\left\langle E^{\prime} \otimes_{K_{0}} K_{1}\right\rangle_{n}$. Therefore $U_{K_{1} / K_{0}}\left(G^{\prime} \otimes_{K_{0}} K_{1}\right)$ belongs to $\left\langle U_{K_{1} / K_{0}}\left(E^{\prime} \otimes_{K_{0}} K_{1}\right)\right\rangle_{n}$. Hence by Lemma 2.1. $G^{\prime}$ belongs to $\left\langle E^{\prime}\right\rangle_{n}$.

(2) We assume that $K / k$ is a separable algebraic extension. We prove that for a perfect $\operatorname{dg}$ - $A$-module $E \in \operatorname{Perf}(A)$ such that $\langle E\rangle_{n}=\operatorname{Perf}(A)$ for some $n \in \mathbb{N}$ we have $\left\langle E \otimes_{k} K\right\rangle_{n}=\operatorname{Perf}\left(A_{K}\right)$.

First we assume that the separable extension $K / k$ is finite dimensional. Let $\bar{G}$ be a perfect $\mathrm{dg}-A_{K}$-module. Since the extension $K / k$ is finite dimensional, $U(\bar{G})$ belongs to $\langle E\rangle_{n}=\operatorname{Perf}(A)$. Hence $U(\bar{G}) \otimes_{k} K$ belongs to $\left\langle E \otimes_{k} K\right\rangle_{n}$. Since the extension $K / k$ is separable, the morphism $\mu: K \otimes_{k} K \rightarrow K, x \otimes y \mapsto x y$ of $K \otimes_{k} K$-modules has a section. Hence the morphism $\bar{G} \otimes_{K} \mu$ of $\operatorname{dg}-A_{K}$-modules has a section

$$
U(\bar{G}) \otimes_{k} K \cong \bar{G} \otimes_{K} K \otimes_{k} K \stackrel{\bar{G} \otimes_{K} \mu}{\longrightarrow} \bar{G} \otimes_{K} K \cong \bar{G} .
$$

Therefore $\bar{G}$ is a direct summand of $U(\bar{G}) \otimes_{k} K$. Hence by Lemma 2.1, $\bar{G}$ belongs to $\left\langle E \otimes_{k} K\right\rangle_{n}$.

Finally we prove the general case. Let $\bar{G}$ be a perfect $\mathrm{dg}-A_{K}$-module. By Lemma 2.2 there exists an intermediate field $k \subset K_{0} \subset K$ which is finite dimensional over $k$ such that there exists a perfect $A_{K_{0}}$-module $G^{\prime}$ such that $G^{\prime} \otimes_{K_{0}} K \cong \bar{G}$ in $\mathcal{D}\left(A_{K}\right)$. Since $G^{\prime}$ belongs to $\left\langle E \otimes_{k} K_{0}\right\rangle_{n}$ by the first step, we conclude that $\bar{G}$ belongs to $\left\langle E \otimes_{k} K\right\rangle_{n}$.

Remark 2.3. (1) If an algebraic extension $K / k$ is not separable, then the dimension tridim $\operatorname{Perf}\left(A_{K}\right)$ is possibly larger than the dimension tridim $\operatorname{Perf}(A)$.

Here is an example. Let $F$ be a field of characteristic $p>0$. Let $K:=F(t)$ be a rational function field in one variable and define $k:=F\left(t^{p}\right) \subset K=F(t)$. Set $A:=K$. Then it is easy to see that $A_{K} \cong K[x] /\left(x^{p}\right)$. Since gldim $A_{K}=\infty$, we see that tridim $\operatorname{Perf}\left(A_{K}\right)=\infty$ by [3, Proposition 7.26]. However since $A=K$ is a field, we have tridim $\operatorname{Perf}(A)=0$.

(2) When the extension $K / k$ is not algebraic, the dimension tridim $\operatorname{Perf}\left(A_{K}\right)$ is possibly larger than tridim $\operatorname{Perf}(A)$ even if an extension $K / k$ is separable.

Here is an example. Assume that for simplicity $k$ is algebraically closed. Let $K=k(y)$ and $A=k(x)$ be rational function fields in one variable over $k$. We claim that tridim $\operatorname{Perf}\left(A_{K}\right)=1$. First note that $A_{K}$ is the localization $S^{-1} k[x, y]$ of the polynomial algebra $k[x, y]$ in two variables by the multiplicative set $S=$ $\{f(x) g(y) \mid f(x) \in k[x] \backslash 0, g(y) \in k[y] \backslash 0\}$. Hence it is easy to see that $A_{K}$ is a regular algebra essentially of finite type over $k$ of dimension 1 . Therefore we see that $\operatorname{Perf}\left(A_{K}\right) \simeq \mathcal{D}^{b}\left(\bmod A_{K}\right)$ and tridim $\operatorname{Perf}\left(A_{K}\right) \leq 1$ by [3, Proposition 7.4$]$. We denote by $W$ the image of the canonical embedding $i$ : Spec $A_{K} \hookrightarrow \operatorname{Spec} k[x, y]$. It is easy to see that for every maximal ideal $\mathfrak{m}$ of $k[x, y]$ there exists a prime ideal $\mathfrak{p}$ of height 1 contained in $\mathfrak{m}$ which belongs to $W$ (for a maximal ideal $\mathfrak{m}=(x-a, y-b)$ where $a, b \in k$, it is enough to set $\mathfrak{p}:=(x+y-a-b))$. Therefore we see that for 
every nonempty open set $U$ of Spec $k[x, y]$, there exists a prime ideal $\mathfrak{p}$ of height 1 which belongs to $U \cap W$. Since the derived pullback functor $\mathbb{R} i^{*}: \mathcal{D}^{b}(\bmod k[x, y]) \rightarrow$ $\mathcal{D}^{b}\left(\bmod A_{K}\right)$ is essentially surjective, by the method of proof of [3, Theorem 7.17 ] we conclude that tridim $\operatorname{Perf}\left(A_{K}\right)=1$. However, since $A=k(x)$ is a field, we see that tridim $\operatorname{Perf}(A)=0$.

\section{Proof of Theorem 1.2}

Proof of Theorem 1.2. We denote the algebraic closure of $k$ by $\bar{k}$. By Lemma 3.1 below it is enough to show that $A_{\bar{k}}:=A \otimes_{k} \bar{k}$ has finite representation type. By [5] it is enough to show that tridim $\underline{\bmod } A_{\bar{k}}=0$. Since we assume that $\operatorname{tridim} \underline{\bmod } A=0$, there exists a finite $A$-module $E$ such that $\langle E\rangle_{1}=\underline{\bmod } A$. By the same method of the proof of Theorem 1.1 (2), we see that $\langle E \otimes \bar{k}\rangle_{1}=\underline{\bmod } A_{\bar{k}}$. This completes the proof of Theorem 1.2 .

Lemma 3.1. Let $A$ be a finite dimensional $k$-algebra. If $A_{\bar{k}}$ is of finite representation type, then $A$ is of finite representation type.

For a finitely generated $A$-module $M$, we denote by add $(M)$ the smallest full subcategory of $\bmod A$ which is closed under taking finite direct sums, direct summands and isomorphisms.

Proof. Assume that $A_{\bar{k}}$ is of finite representation type. There exists a finitely generated $A_{\bar{k}}$-module $\bar{N}$ such that add $\bar{N}=\bmod A_{\bar{k}}$. Since $\bar{N}$ has a finite presentation $A_{\bar{k}}^{\oplus n} \rightarrow A_{\bar{k}}^{\oplus m} \rightarrow \bar{N} \rightarrow 0$, by the same method as in the proof of Lemma 2.2, we see that there exists an intermediate field $k \subset K_{0} \subset \bar{k}$ which is finite dimensional over $k$ such that there exists a finitely generated $A_{K_{0}}$-module $N^{\prime}$ such that $N^{\prime} \otimes_{K_{0}} \bar{k} \cong \bar{N}$ as $A_{\bar{k}}$-modules. We prove that $\operatorname{add}\left(U\left(N^{\prime}\right)\right)=\bmod A$ where $U=U_{K_{0} / k}: \bmod A_{K_{0}} \rightarrow \bmod A$ is the forgetful functor. Let $M$ be a finitely generated $A$-module. Since $M \otimes_{k} \bar{k}$ belongs to add $\left(N^{\prime} \otimes_{K_{0}} \bar{k}\right)$, by the same method as in the proof of Lemma 2.2, we see that there exists an intermediate field $K_{0} \subset K_{1} \subset \bar{k}$ which is finite dimensional over $k$ such that $M \otimes_{k} K_{1}$ belongs to $\operatorname{add}\left(N^{\prime} \otimes_{K_{0}} K_{1}\right)$. Therefore we have $\operatorname{add}\left(M \otimes_{k} K_{1}\right) \subset \operatorname{add}\left(N^{\prime} \otimes_{K_{0}} K_{1}\right)$. Let $U^{\prime}:=U_{K_{1} / k}: \bmod A_{K_{1}} \rightarrow \bmod A$ be the forgetful functor. By the same method as in the proof of Lemma 2.1, we see that $\operatorname{add}(M)=\operatorname{add}\left(U^{\prime}\left(M \otimes_{k} K_{1}\right)\right)$ and that $\operatorname{add}\left(U\left(N^{\prime}\right)\right)=\operatorname{add}\left(U^{\prime}\left(N^{\prime} \otimes_{K_{0}} K_{1}\right)\right)$. Hence $M$ belongs to $\operatorname{add}\left(U\left(N^{\prime}\right)\right)$.

\section{ACKNOWLEDGEMEnts}

The author thanks O. Iyama for suggesting the example in Remark 2.3(1). He also thanks H. Ikoma, S. Takagi and M. Yoshiwaki for valuable discussions. The author was supported by Grant-in-Aid for Young Scientists (B)21740017.

\section{REFERENCES}

[1] Marcel Bökstedt and Amnon Neeman, Homotopy limits in triangulated categories, Compositio Math. 86 (1993), no. 2, 209-234. MR1214458 (94f:18008)

[2] Dieter Happel, Triangulated categories in the representation theory of finite-dimensional algebras, London Mathematical Society Lecture Note Series, vol. 119, Cambridge University Press, Cambridge, 1988. MR935124 (89e:16035) 
[3] Raphaël Rouquier, Dimensions of triangulated categories, J. K-Theory 1 (2008), no. 2, 193256, DOI 10.1017/is007011012jkt010. MR2434186(2009i:18008)

[4] Raphaël Rouquier, Representation dimension of exterior algebras, Invent. Math. 165 (2006), no. 2, 357-367, DOI 10.1007/s00222-006-0499-7. MR2231960(2007f:16031)

[5] M. Yoshiwaki. On self-injective algebras of stable dimension zero, Nagoya Math. J. 203 (2011), 101-108. MR2834250 (2012h:16009)

Research Institute for Mathematical Sciences, Kyoto University, Kyoto 606-8502, JAPAN

E-mail address: minamoto@kurims.kyoto-u.ac.jp 